\title{
Inversion-based Synthesis Algorithm of Periodic Fault Detection and Isolation Filters*
}

\author{
A. Varga
}

\begin{abstract}
A numerically reliable lifting-free computational method is proposed to solve fault detection and isolation problems for periodic systems using a model matching approach. The synthesis procedure employs numerically reliable algorithms to determine least order annihilators of periodic systems to reduce the corresponding periodic model matching problem to a simpler form. The reduced problem is then solved using an explicit periodic system inversion based approach. If the resulting fault detection filter is not stable and/or not causal, then a final stabilization step is performed using periodic coprime factorization techniques. The proposed integrated synthesis algorithm has strongly coupled computational steps, where all available structural information at the end of each computational step are fully exploited in the subsequent computations.
\end{abstract}

\section{INTRODUCTION}

The solution of the periodic fault detection and isolation problem (PFDIP) has its main application in solving fault isolation problems for multirate systems. There are several possible approaches to solve PFDIP. In what follows, we give a short account of existing techniques.

A straightforward approach for the solution of the PFDIP is to employ methods proposed for solving fault detection and isolation problems for linear time-invariant (LTI) systems. This approach is in principle simple and relies on building a lifted LTI model which is input-output equivalent to the given linear time-periodic (LTP) system [1], [2]. Then, suitable linear synthesis methods can be applied to the lifted LTI model, as for example, determining a bank of LTI filters which provides a structured residual set [3], or solving a model matching problem which results by imposing appropriate structural conditions [4]. Numerically reliable computational methods for this purpose are described in a recent survey paper [5]. The final step consists in recovering a periodic realization of the fault detection filter which can be used for real-time implementations.

Several intrinsic difficulties can impede the usage of lifting-based approaches, especially for systems with high orders or large periods. For example, building a lifted representation using the lifting technique of [1] involves explicitly forming many matrix products, thus this approach is completely unappropriate from numerical point of view. On the other hand, using the lifting technique proposed in [2] requires manipulating large sparse matrices of a descriptor system representation, which leads to computationally unacceptable costs. Even the final step of turning the designed

A. Varga is with the German Aerospace Center, DLR - Oberpfaffenhofen, Institute of System Dynamics and Control, D-82234 Wessling, Germany Andreas.Varga@dlr.de lifted representation of the detector into a periodic state space representation (e.g., by using the algorithm of [6]) can lead to numerical difficulties in the case of high order systems. Therefore, lifting-free methods should be always preferred in solving computational problems for periodic systems [7].

Methods for solving the periodic fault detection problem (PFDP) can be employed to solve the PFDIP, similarly as for the LTI case, by synthesizing a bank of periodic fault detection filters, which provides a structured residual set. The synthesis method proposed in [8] uses a periodic parity space approach and is able to produce fault detection filters of least possible orders. The main weakness of this approach is the need to form matrix powers and matrix products to perform rank determinations. Therefore, from a numerical point of view this method is potentially not reliable. A lifting-free geometric approach for the synthesis of fault detection filters has been developed in [9], using a statespace synthesis model without direct feedthrough terms. The proposed synthesis procedure determines an unknown-input observer (UIO) of the same size as the initial system. The main limitation of this approach is that a necessary condition for the existence of a stable UIO is that the system formed only with the disturbance inputs must not have unstable zeros [10]. Thus, although a stable fault detection filter may exist, there may exist no stable UIO which can serve as a fault detection filter. The approach of [9] has been extended in [11] to solve the PFDIP, by designing a bank of fault detection filters which provide a structured set of residuals. Since both in [9] and [11] the least order synthesis aspect has not been addressed, each of these filters has the order of the original system, and therefore the resulting global fault detection and isolation filter has potentially an unnecessary high order. Recently, a similar lifting-free synthesis approach has been proposed in [12], which relies exclusively on reliable numerical techniques. The resulting partial detectors have least dynamical orders and thus the overall order of detector can be kept relatively low.

In this paper we propose a new lifting-free approach to solve the PFDIP, which relies on numerically reliable algorithms closely tied in an integrated synthesis method, whose successive synthesis steps provide partial solutions of the PFDIP. The proposed approach can be seen as an extension to the periodic case of the inversion-based synthesis technique proposed in [5] for standard systems. In a similar way, two main computational steps are performed: 1) the determination of a periodic left annihilator to decouple the input and disturbance inputs in the residuals; this allows to reduce the initial problem to a simpler one, and, 2) the 
solution of a regular periodic model matching problem using a system inversion based approach. An important feature of the proposed method is its ability to determine or update a reference model used to solve the synthesis problem. The main computational ingredients are algorithms for the computation of periodic annihilators [13], minimal periodic dynamic covers [14] and periodic coprime factorizations [15].

Notation. For an $N$-periodic matrix $X_{k}$ we use alternatively the script notation $\mathcal{X}:=\operatorname{diag}\left(X_{1}, X_{2}, \ldots, X_{N}\right)$, which associates the block-diagonal matrix $\mathcal{X}$ to the cyclic matrix sequence $X_{k}, k=1, \ldots, N$.

\section{Periodic Fault Detection And Isolation PROBLEM}

We consider periodic time-varying linear discrete-time descriptor systems of the form

$$
\begin{aligned}
E_{k} x(k+1) & =A_{k} x(k)+B_{k}^{u} u(k)+B_{k}^{d} d(k)+B_{k}^{f} f(k) \\
y(k) & =C_{k} x(k)+D_{k}^{u} u(k)+D_{k}^{d} d(k)+D_{k}^{f} f(k)
\end{aligned}
$$

where, for generality, the system state vector is assumed to have time-varying dimensions $x(k) \in \mathbf{R}^{n_{k}}, y(k) \in \mathbf{R}^{p}$ is the measured output vector, $u(k) \in \mathbf{R}^{m_{u}}$ is the plant control input vector, $d(k) \in \mathbf{R}^{m_{d}}$ is the disturbance vector, and $f(k) \in \mathbf{R}^{m_{f}}$ is the fault signal vector. We assume that the system matrices $E_{k}, A_{k}, B_{k}^{u}, \ldots$ are periodic with period $N \geq 1$ and $E_{k}$ is square for $k=1, \ldots, N$. For invertible $E_{k}$ the periodic system is causal, and often we can employ standard realizations with $E_{k}=I_{n_{k+1}}$ (i.e., the identity matrix of appropriate size). If $E_{k}$ is singular, then the periodic system may be in general non-causal. Periodic descriptor systems as in (1) will be alternatively denoted by a quintuple of periodic matrices $\left(E_{k}, A_{k}, B_{k}, C_{k}, D_{k}\right)$ or by a quadruple $\left(A_{k}, B_{k}, C_{k}, D_{k}\right)$ in the standard case, with appropriately defined $B_{k}$ and $D_{k}$.

The periodic fault detection and isolation problem (PFDIP) can be formulated as follows: Determine a stable $N$-periodic linear residual generator having the general form

$$
\begin{aligned}
\widehat{x}(k+1) & =F_{k} \widehat{x}(k)+H_{k}\left[\begin{array}{c}
y(k) \\
u(k)
\end{array}\right] \\
r(k) & =J_{k} \widehat{x}(k)+L_{k}\left[\begin{array}{c}
y(k) \\
u(k)
\end{array}\right]
\end{aligned}
$$

such that for all control and disturbance inputs $u(k)$ and $d(k)$, and for $i=1, \ldots, m_{f}$

(i) $r_{i}(k)=0$ if $f_{i}(k)=0$, (decoupling condition)

(ii) $r_{i}(k) \neq 0$ if $f_{i}(k) \neq 0$, (fault isolation condition)

where $k \geq 0$ and for $(i)$ and $(i i)$ we assume zero initial conditions for the state variables: $x(0)=0$ and $\widehat{x}(0)=0$.

The stability requirement can be expressed by the condition that all characteristic multipliers of the periodic matrix $F_{k}$ (i.e., the eigenvalues of the monodromy matrix $\Psi_{F}:=$ $F_{N} \cdots F_{2} F_{1}$ ) have moduli less than one [16]. More generally, the stability requirement can be formulated with respect to a good symmetric region $\mathbb{C}_{g}$ of the unit disk centered in the origin, by requiring that the spectrum of $\Psi_{F}$ satisfies $\Lambda\left(\Psi_{F}\right) \subset \mathbb{C}_{g}$.
To derive algebraic conditions for the solution of the PFDIP, we reformulate the detector design problem in terms of the transfer-function matrix (TFM) corresponding to the associated stacked lifted representation of [2], which uses the input-state-output behavior of the system over time intervals of length $N$, rather than 1 . The lifted input, output and state vectors are defined as

$$
\begin{aligned}
\widetilde{u}(h) & =\left[u^{T}(h N+1) \cdots u^{T}(h N+N)\right]^{T}, \\
\widetilde{d}(h) & =\left[d^{T}(h N+1) \cdots d^{T}(h N+N)\right]^{T}, \\
\widetilde{f}(h) & =\left[f^{T}(h N+1) \cdots f^{T}(h N+N)\right]^{T}, \\
\widetilde{y}(h) & =\left[y^{T}(h N+1) \cdots y^{T}(h N+N)\right]^{T}, \\
\widetilde{x}(h) & =\left[x^{T}(h N+1) \cdots x^{T}(h N+N)\right]^{T} .
\end{aligned}
$$

and the corresponding lifted system can be represented by a LTI descriptor system of the form (notice the usage of script notation)

$$
\begin{aligned}
E^{S} \widetilde{x}(h+1) & =A^{S} \widetilde{x}(h)+\mathcal{B}^{u} \widetilde{u}(h)+\mathcal{B}^{d} \widetilde{d}(h)+\mathcal{B}^{f} \widetilde{f}(h) \\
\widetilde{y}(h) & =\mathcal{C} \widetilde{x}(h)+\mathcal{D}^{u} \widetilde{u}(h)+\mathcal{D}^{d} \widetilde{d}(h)+\mathcal{D}^{f} \widetilde{f}(h)
\end{aligned},
$$

where the pole pencil corresponding to the periodic pair $\left(A_{k}, E_{k}\right)$

$$
A^{S}-z E^{S}=\left[\begin{array}{ccccc}
A_{1} & -E_{1} & O & \cdots & O \\
O & \ddots & \ddots & \ddots & \vdots \\
\vdots & \ddots & \ddots & -E_{N-2} & O \\
O & & \ddots & A_{N-1} & -E_{N-1} \\
-z E_{N} & O & \cdots & O & A_{N}
\end{array}\right]
$$

is regular. For the lifted system (3), the TFMs $G_{u}(z), G_{d}(z)$, $G_{f}(z)$ from the control, disturbance, and fault inputs to the system output are

$$
G_{\xi}(z)=\mathcal{C}\left(z E^{S}-A^{S}\right)^{-1} \mathcal{B}^{\xi}+\mathcal{D}^{\xi}
$$

where $\xi$ stays for $u, d$ and $f$, respectively.

The representation (2) is termed in accordance with [3] the implementation form of the periodic residual generator. Assume that the residual generator (2) has a lifted representation with the corresponding TFM $Q(z)$. Let $R_{u}(z), R_{d}(z)$ and $R_{f}(z)$ be the corresponding TFMs from the control, disturbance and fault inputs to the residual given by

$\left[R_{u}(z) R_{d}(z) R_{f}(z)\right]=Q(z)\left[\begin{array}{ccc}G_{u}(z) & G_{d}(z) & G_{f}(z) \\ I_{N m_{u}} & 0 & 0\end{array}\right]$

The periodic realization corresponding to the above lifted TFMs is termed according to [3] the internal form of the residual generator.

For the PFDIP, the decoupling condition $(i)$ requires $R_{u}(z)=0$ and $R_{d}(z)=0$, or equivalently

$$
Q(z) G(z)=0
$$

where

$$
G(z)=\left[\begin{array}{cc}
G_{u}(z) & G_{d}(z) \\
I_{N m_{u}} & O
\end{array}\right]
$$

The fault isolation condition $(i i)$ is equivalent to ask $R_{f}(z)=M_{r}(z)$ or equivalently

$$
Q(z)\left[\begin{array}{c}
G_{f}(z) \\
0
\end{array}\right]=M_{r}(z)
$$


where $M_{r}(z)$ is the lifted TFM of a suitable reference model describing the desired dependence of the residuals of faults. In general, $M_{r}(z)$ corresponds to $m_{f}$ diagonally stacked single-input single-output (SISO) stable periodic systems. The conditions (6) and (8) for a given $M_{r}(z)$ leads to a model-matching formulation of the PFDIP, which we aim to solve achieving the least possible order of the state-space realization of the periodic filter with the TFM $Q(z)$.

The synthesis conditions (6) and (8) must be complemented with the requirement of stability for $Q(z)$. However, the actual choice of $M_{r}(z)$ may lead to a solution $Q(z)$ which corresponds to a non-causal and/or unstable periodic system. Therefore, besides determining $Q(z)$, we also consider the determination of a suitable updating factor $M(z)$ of $M_{r}(z)$ to ensure the stability and causality of the solution $Q(z)$ satisfying $R_{f}(z)=M(z) M_{r}(z)$. Obviously, $M(z)$ must be chosen such that it corresponds to a causal, stable and invertible periodic system. Additionally, $M(z)$ must be chosen to have the same (block diagonal) structure as $M_{r}(z)$.

A necessary and sufficient condition for the existence of a solution to the PFDIP can be derived from those for LTI systems [17]:

Theorem 1: Given the $N m_{f} \times N m_{f}$ lifted TFM of an invertible reference model $M_{r}(z)$, the PFDIP is solvable for the periodic system (1) if and only if

$$
\operatorname{rank}\left[G_{d}(z) G_{f}(z)\right]=\operatorname{rank} G_{d}(z)+N m_{f}
$$

The reference model $M_{r}(z)$ can be a given periodic system with $m_{f}$ inputs and $m_{f}$ outputs, corresponding to $m_{f}$ diagonally stacked SISO periodic systems. In this case, the PFDIP can be formulated as a periodic model matching problem (PMMP) to determine a stable solution $Q(z)$ (whenever one exists) of the the equations (6) and (8). Alternatively, the synthesis procedure of the filter $Q(z)$ can be used to determine a suitable $M_{r}(z)$ with the above mentioned properties which guarantees the stability of $Q(z)$. In the next section we propose a new synthesis algorithm of a residual generator (2) which solves the PFDIP by solving a PMMP. In Section IV we present a lifting-free version based on explicit periodic state-space representations.

\section{INVERSION-BASED SOLUTION OF THE PFDIP}

As basis for our synthesis procedure, we extend an algorithm recently proposed for LTI systems in [5] to LTP systems. The proposed integrated synthesis approach relies on repeated updating of an initial fault detection filter. The final filter results in a factored form with an explicitly determined periodic state space realization. For more clarity, we describe first the main steps of this algorithm in terms of the TFMs of the lifted representation. In the next section, we describe the computational variant of this algorithm in terms of the original periodic representation (1).

\section{Step 1. Nullspace based reduction}

If $m_{u}+m_{d}>0$, we choose $Q(z)$ in a factored form

$$
Q(z)=\bar{Q}_{1}(z) Q_{1}(z)
$$

where $Q_{1}(z)$ is a proper left rational nullspace basis satisfying $Q_{1}(z) G(z)=0$ and $\bar{Q}_{1}(z)$ is a factor to be subsequently determined. With this choice, it follows that $Q(z)$ automatically fulfills the decoupling conditions in $(i)$, namely, $R_{u}(z)=0$ and $R_{d}(z)=0$. The resulting $Q_{1}(z)$ has maximal row rank $N p-r_{d}$, where $r_{d}=\operatorname{rank} G_{d}(z)$. Thus, the existence condition of a nonempty rational nullspace basis $Q_{1}(z)$ is simply $r_{d}<N p$. This condition is guaranteed provided the existence conditions of Theorem 1 are fulfilled.

With the above detector, the PMMP has been reduced to solving

$$
\bar{Q}_{1}(z) \bar{G}_{f}(z)=M_{r}(z),
$$

with

$$
\bar{G}_{f}(z):=Q_{1}(z)\left[\begin{array}{c}
G_{f}(z) \\
0
\end{array}\right]
$$

Note that we can always choose $Q_{1}(z)$ to correspond to a causal and stable periodic system and such that $\bar{G}_{f}(z)$ defined in (12) corresponds to a causal and stable periodic system as well [13].

If $m_{u}=m_{d}=0$, we can determine $Q_{1}(z)$ simply from a left coprime factorization

$$
G_{f}(z)=Q_{1}^{-1}(z) \bar{G}_{f}(z)
$$

such that $Q_{1}(z)$ and $\bar{G}_{f}(z)$ correspond to causal and stable periodic systems. Suitable state space algorithms for this purpose are described in [18].

With this first preprocessing step, we reduced the original PMMP formulated for the periodic system (1) to one formulated for a reduced periodic system with the lifted TFM $\bar{G}_{f}(z)$ and without control and disturbance inputs. For this system, we have to determine the TFM $\bar{Q}_{1}(z)$ which solves the reduced PMMP corresponding to (11). The solvability condition (9) of Theorem 1 reduces to the left invertibility condition for $\bar{G}_{f}(z)$, as given in the next corollary.

Corollary 1: Given the $N m_{f} \times N m_{f}$ lifted TFM of an invertible reference model $M_{r}(z)$, the PFDIP is solvable for the periodic system (1) if and only if

$$
\operatorname{rank} \bar{G}_{f}(z)=N m_{f}
$$

\section{Step 2. Regularization}

We can choose $\bar{Q}_{1}(z)$ in the form

$$
\bar{Q}_{1}(z)=\bar{Q}_{2}(z) Q_{2}(z),
$$

where $\bar{Q}_{2}(z)$ is still to be determined and $Q_{2}(z)$ is a prefilter chosen such that

$$
\widetilde{G}_{f}(z):=Q_{2}(z) \bar{G}_{f}(z)
$$

is invertible. The simplest choice of $Q_{2}(z)$ is a constant projection matrix which simply selects $N m_{f}$ linearly independent rows of $\bar{G}_{f}(z)$. A more involved choice is one leading to a full row rank (thus invertible) $\widetilde{G}_{f}(z)$ and simultaneously to $Q_{2}(z) Q_{1}(z)$ having least dynamical order. Such a choice is possible using periodic minimal dynamic cover techniques [19] (see Section IV). 
Step 3. Inversion-based updating

We choose $\bar{Q}_{2}(z)$ in the form

$$
\bar{Q}_{2}(z)=Q_{4}(z) Q_{3}(z),
$$

where $Q_{4}(z)$ is still to be determined (see next step) and

$$
Q_{3}(z)=M_{r}(z) \widetilde{G}_{f}^{-1}(z) .
$$

\section{Step 4. Stabilization}

If $M_{r}(z)$ has been chosen to guarantee the cancellation of all unstable poles of $Q_{3}(z) Q_{2}(z) Q_{1}(z)$, then we can choose $Q_{4}(z)$ simply the identity matrix of order $N m_{f}$. In the general case, we choose $Q_{4}(z)=M(z)$, where $M(z)$ ensures that the resulting final detector with the lifted TFM

$$
Q(z)=Q_{4}(z) Q_{3}(z) Q_{2}(z) Q_{1}(z),
$$

corresponds to a stable and causal periodic system realization. $M(z)$ can be determined using stable and proper coprime factorization techniques (see Section IV) and can be interpreted as an updating factor for $M_{r}(z)$.

This synthesis method we call the inversion based method and extends the inversion method proposed for LTI systems in [5]. For numerical computations, an integrated synthesis algorithm is described in the next section.

\section{Computational algorithms}

In this sections, we present an equivalent synthesis approach for periodic systems without manipulating explicitly lifted representations. The proposed computational approach operates directly on the matrices of the original periodic state-space description (1) and computes left annihilators directly in periodic minimal state-space representations. All subsequent computations to determine a stable fault detection filter which solves the PFDIP can be interpreted as updates of the initial representation and can be done using reliable numerical techniques based on state-space computations.

\section{Step 1. Computation of a maximal left annihilator}

In this computational step we employ the computational approach of [13] to determine a maximal left annihilator with the lifted TFM $Q_{1}(z)$ for the periodic system

$$
\begin{aligned}
E_{k} x(k+1) & =A_{k} x(k)+B_{k}^{u} u(k)+B_{k}^{d} d(k) \\
{\left[\begin{array}{c}
y(k) \\
u(k)
\end{array}\right] } & =\left[\begin{array}{c}
C_{k} \\
0
\end{array}\right] x(k)+\left[\begin{array}{c}
D_{k}^{u} \\
I_{m_{u}}
\end{array}\right] u(k)+\left[\begin{array}{c}
D_{k}^{d} \\
0
\end{array}\right] d(k),
\end{aligned}
$$

which corresponds to the lifted TFM $G(z)$ in (7). In terms of lifted representations, this amounts to determine a periodic system (e.g., of the form (2)), whose lifted TFM $Q_{1}(z)$ is a proper rational matrix whose rows form a rational basis for the left nullspace of $G(z)$ (i.e., $Q_{1}(z) G(z)=0$ ) see, for example, [20].

According to [13], $Q_{1}(z)$ can be determined to have a causal periodic realization of the form

$$
\begin{aligned}
E_{k}^{l} \bar{x}(k+1) & =A_{k}^{l} \bar{x}(k)+B_{k}^{l}\left[\begin{array}{c}
y(k) \\
u(k)
\end{array}\right] \\
\bar{y}(k) & =C_{k}^{l} \bar{x}(k)+D_{k}^{l}\left[\begin{array}{c}
y(k) \\
u(k)
\end{array}\right]
\end{aligned}
$$

with nonsingular $E_{k}^{l}$. Note that, this detector realization is observable, and is obtained in general with time-varying dimensions of the state vector $\left(\bar{n}_{k}\right)$ and output vector $\left(q_{k}\right)$. The output vector dimensions sum up to $\sum_{k=1}^{N} q_{k}=N p-$ $r_{d}$. Generically, $r_{d}=N r_{d}^{\prime}$, where $r_{d}^{\prime}=\operatorname{rank} G_{d}(z) / N$. Therefore, $q_{k}=p-r_{d}^{\prime}$ and thus constant for all $k$.

A realization of

$$
\bar{G}_{f}(z):=Q_{1}(z)\left[\begin{array}{c}
G_{f}(z) \\
0
\end{array}\right]
$$

can be obtained in the form

$$
\begin{aligned}
E_{k}^{l} x_{f}(k+1) & =A_{k}^{l} x_{f}(k)+\widetilde{B}_{k}^{f} f(k) \\
r(k) & =C_{k}^{l} x_{f}(k)+\widetilde{D}_{k}^{f} f(k)
\end{aligned}
$$

As it can be observed, the realizations of $Q_{1}(z)$ and $\bar{G}_{f}(z)$ share the matrices $E_{k}^{l}, A_{k}^{l}$, and $C_{k}^{l}$.

To determine the left annihilator (20), a single reduction of a periodic pair to a periodic Kronecker-like form has to be performed using the algorithm of [21]. This algorithm performs exclusively orthogonal transformations on a pair of periodic matrices, and it is possible to easily prove that all computed matrices are exact for a slightly perturbed original system. It follows that the algorithm to compute the left annihilator (20) and the corresponding realization of $\bar{G}_{f}(z)$ in (22) is numerically stable.

To check the left invertibility condition (14) we can apply the numerically stable algorithm of [21] to compute the periodic Kronecker-like form of the periodic pair

$$
\left(\left[\begin{array}{cc}
A_{k}^{l} & \widetilde{B}_{k}^{f} \\
C_{k}^{l} & \widetilde{D}_{k}^{f}
\end{array}\right],\left[\begin{array}{cc}
E_{k}^{l} & 0 \\
0 & 0
\end{array}\right]\right)
$$

and check the absence of right structure. Alternatively, the so-called fast algorithms based on orthogonal reductions (see [22]) can be employed to check the full column rank of the system pencil of the lifted system associated to (22).

\section{Step 2. Regularization}

We choose $Q_{2}(z)$ such that $\widetilde{G}_{f}(z):=Q_{2}(z) \bar{G}_{f}(z)$ is invertible. This choice is always possible if the PFDIP is solvable, which is equivalent to $\widetilde{G}_{f}(z)$ being left invertible. If $N p-r_{d}=N m_{f}$, we can use the trivial choice $Q_{2}(z)=$ $I_{N m_{f}}$, while in the case when $N p-r_{d}>N m_{f}$ we can always choose

$$
Q_{2}(z)=\operatorname{diag}\left\{H_{1}, H_{2}, \ldots, H_{N}\right\},
$$

where $H_{1}, H_{2}, \ldots, H_{N}$ are $m_{f} \times q_{k}$ matrices with orthonormal rows.

We can also determine $Q_{2}(z)$ as a prefilter with a periodic realization $\left(E_{k}^{l}, A_{k}^{l}+K_{k} C_{k}^{l}, K_{k}, H_{k} C_{k}^{l}, H_{k}\right)$, where $H_{k}$ and $K_{k}$ are $N$-periodic matrices determined such that $Q_{2}(z) \bar{G}_{f}(z)$ is invertible and $Q_{2}(z) Q_{1}(z)$ has least possible McMillan degree. It is straightforward to show, that the lifted TFM $Q_{2}(z) Q_{1}(z)$ has the periodic state space realization $\left(E_{k}^{l}, A_{k}^{l}+K_{k} C_{k}^{l}, B_{k}^{l}+K_{k} D_{k}^{l}, H_{k} C_{k}^{l}, H_{k} D_{k}^{l}\right)$. For a suitable choice of a periodic $H_{k}$, the main computation is to determine the periodic $K_{k}$ which makes this realization maximally unobservable. The computation of $K_{k}$ can be 
performed using recently developed minimal dynamic cover techniques for periodic systems [19].

The computational details for this computation are presented in [19] (see also [12] for a shorter account). The resulting realizations of $Q_{2}(z) Q_{1}(z)$ of least McMillan degree can be obtained as a minimal state space realization

$$
\left(\widehat{E}_{k}, \widehat{A}_{k}, \widehat{B}_{k}, \widehat{C}_{k}, \widehat{D}_{k}\right) \text {, }
$$

with $\widehat{E}_{k}$ invertible. The periodic realization of the resulting $\widehat{G}_{f}(z):=Q_{2}(z) \bar{G}_{f}(z)$ can be computed in the form

$$
\left(\widehat{E}_{k}, \widehat{A}_{k}, \widehat{B}_{k}^{f}, \widehat{C}_{k}, \widehat{D}_{k}^{f}\right)
$$

Once again, the realizations of $Q_{2}(z) Q_{1}(z)$ and $\widehat{G}_{f}(z)$ share the same matrices $\widehat{E}_{k}, \widehat{A}_{k}$, and $\widehat{C}_{k}$, a property which is instrumental in performing the next computational step.

The choice of suitable $H_{k}$ is straightforward and exploits the fine structure of the periodic pair $\left(A_{k}^{l}, C_{k}^{l}\right)$, which resulted in the previous step in a periodic observability staircase form [23]. For example, we can choose each $H_{k}$ as a $m_{f} \times q_{k}$ matrix with $m_{f}$ leading nonzero columns (e.g., forming a $m_{f} \times m_{f}$ orthogonal matrix), which is equivalent to build $m_{f}$ linear combinations of $m_{f}$ annihilators which correspond to the nonzero columns of $H_{k}$. The cover algorithm of [19] determines suitable periodic transformation matrices such that the choice of a suitable $K_{k}$ is straightforward. The main appeal of this algorithm is that the realizations (23) and (24) result automatically, without the need of a performing additional computations (e.g., minimal periodic realization).

\section{Step 3. Inversion-based updating}

With $Q_{3}(z)=M_{r}(z) \widehat{G}_{f}^{-1}(z)$, we have to compute the updated filter $\bar{Q}(z):=Q_{3}(z) Q_{2}(z) Q_{1}(z)$. We perform this operation in two steps. First, we determine a realization of $\widehat{G}_{f}^{-1}(z) Q_{2}(z) Q_{1}(z)$, which can be obtained explicitly as the periodic descriptor system realization (see Appendix)

$$
\left(\left[\begin{array}{cc}
\widehat{E}_{k} & 0 \\
0 & 0
\end{array}\right],\left[\begin{array}{cc}
\widehat{A}_{k} & \widehat{B}_{k}^{f} \\
\widehat{C}_{k} & \widehat{D}_{k}^{f}
\end{array}\right],\left[\begin{array}{l}
\widehat{B}_{k} \\
\widehat{D}_{k}
\end{array}\right],\left[0-I_{m_{f}}\right], 0\right)
$$

This realization may correspond to a non-causal and/or unstable realization (i.e., with infinite and/or unstable characteristic multipliers). With a proper choice of $M_{r}(z)$, these characteristic multipliers can be cancelled in the realization of $\bar{Q}(z)$. Assuming $M_{r}(z)$ has a standard periodic realization $\left(A_{k}^{r}, B_{k}^{r}, C_{k}^{r}, D_{k}^{r}\right)$, the realization of $M_{r}(z) \widehat{G}_{f}^{-1}(z) Q_{2}(z) Q_{1}(z)$ can be built using standard series coupling formulas of periodic descriptor systems. To achieve the expected poles-zeros cancellations, we apply the algorithm of [14] based on periodic orthogonal similarity transformations to compute an irreducible realization of $\bar{Q}(z)$. Observe that at this moment we have $R_{f}(z)=M_{r}(z)$ for $Q(z)=\bar{Q}(z)$. Therefore, if the resulting filter $\bar{Q}(z)$ is stable, then $Q(z)=\bar{Q}(z)$ is the required solution.

In the case when $\bar{Q}(z)$ is not causal or not stable, we have to perform the additional stabilization step to determine the updating factor $M(z)$ of $M_{r}(z)$, which ensures the stability of the final fault detection filter.

\section{Step 4. Stabilization}

Assume that $\left(\bar{E}_{k}, \bar{A}_{k}, \bar{B}_{k}, \bar{C}_{k}, \bar{D}_{k}\right)$ is the periodic realization of $\bar{Q}(z)$ as resulted at the previous step (e.g., after applying the minimal realization algorithm of [14]). Since the updating factor $M(z)$ must correspond to $m_{f}$ diagonally stacked SISO stable periodic systems, we have

$$
M(z)=\operatorname{diag}\left\{M_{1}(z), M_{2}(z), \ldots, M_{m_{f}}(z)\right\},
$$

where $M_{j}(z)$ is the lifted TFM of the $j$-th SISO periodic subsystem. In what follows, we describe the computation of a minimal realization of $M_{j}(z)$ and of the corresponding block rows $Q_{j}(z):=M_{j}(z) \bar{Q}_{j}(z)$, where $\bar{Q}_{j}(z)$ is the block row of $\bar{Q}(z)$ corresponding to the $j$-th filter output.

Let $\bar{C}_{k, j}$ and $\bar{D}_{k, j}$ denote the $j$-th rows of $\bar{C}_{k}$ and $\bar{D}_{k}$, respectively. Thus, $\left(\bar{E}_{k}, \bar{A}_{k}, \bar{B}_{k}, \bar{C}_{k, j}, \bar{D}_{k, j}\right)$ is a realization of $\bar{Q}_{j}(z)$ which may be non-minimal. A minimal realization can be computed using the algorithm of [14]. For simplicity, we reuse the same notation for the resulting minimal realization.

If the periodic pair $\left(\bar{E}_{k}, \bar{A}_{k}\right)$ has infinite characteristic multipliers, then we employ the dual of the CPRCF Algorithm of [18] to compute a stable causal periodic left coprime factorization

$$
\bar{Q}_{j}(z)=M_{j}^{-1}(z) Q_{j}(z)
$$

where both $M_{j}(z)$ and $Q_{j}(z)$ correspond to stable causal periodic systems. If the realization of $\bar{Q}_{j}(z)$ is causal, then the PLCF Algorithm of [18] can be employed to compute a stable periodic left coprime factorization (PLCF) of $\bar{Q}_{j}(z)$ as above. Both above algorithms are numerically reliable and rely on sound computational techniques, as the computation of left periodic annihilators of periodic systems and coprime factorization using recursive generalized periodic Schur technique for the assignment of characteristic multipliers.

\section{Discussion AND CONCLUSIONS}

A typical modular approach to solve various fault detection problems can be described as a composition of modularized computational steps, where the problem structure between steps is often not exploited. In contrast, an integrated approach consists of computational steps which are connected at a finer granularity level by exploiting all structural information at the termination of each step. The expected advantages of an integrated approach are: more efficient computations with less overhead and less storage, and also an increased computational reliability, because the structural aspects can be fully exploited.

The proposed numerical solution of the PFDIP is an example of a numerically reliable integrated computational algorithm, with closely connected computational steps. The fault detection filter is implicitly determined in a factored form, where each factor corresponds to a typical computational step. In a modular approach, the explicit computation of these factors would lead to high order detector models which are usually non-minimal, and therefore an additional minimal realization step is necessary. In contrast, the proposed integrated synthesis approach relies on fault detection 
filter updating techniques, where implicit cancellations are performed by determining explicit minimal order state space realizations. In the proposed approach, a left annihilator with the lifted TFM $Q_{1}(z)$ serves for the initialization of the updating process, $Q(z)=Q_{1}(z)$, while at the successive computational steps $Q(z)$ is updated: $Q(z) \leftarrow Q_{i}(z) Q(z)$, for $i=2, \ldots, 4$. In a similar way, the updating of the internal form of the detector is performed in the first two steps, where only the nonzero TFMs $R_{f}(z)$ has to be updated, because the initial choice already guarantees $R_{u}(z)=0$ and $R_{d}(z)=0$.

Typical examples illustrating the close coupling between steps are the regularization and inversion steps. In the regularization step, the synthesis fully exploits the staircase structure of the periodic pairs $\left(A_{k}^{l}, C_{k}^{l}\right)$ in choosing appropriate linear combinations via the matrices $H_{k}$. Since both the implementation and internal forms of the detector are simultaneously updated, they have the same order and share a part of the state space matrices. This allows to obtain an explicit state space realization of the intermediary detector $\widehat{G}_{f}^{-1}(z) Q_{2}(z) Q_{1}(z)$ in the inversion step (see Appendix).

For all computational steps, numerically reliable computational algorithms are available. These algorithms satisfy the requirements for a satisfactory algorithm for periodic systems as formulated in [7]. The algorithms to compute periodic annihilators or to perform periodic minimal realizations are numerically stable, by relying exclusively on orthogonal transformations. The algorithms for periodic dynamic covers and coprime factorizations perform both orthogonal but also non-orthogonal transformations. These algorithms are still numerically reliable, because the potential loss of accuracy can be easily detected. Therefore, the overall synthesis algorithm can be considered a numerically reliable approach.

\section{APPENDIX}

$$
\text { Periodic realization of } \widehat{G}_{f}^{-1}(z) Q_{2}(z) Q_{1}(z)
$$

Consider the periodic state-space realizations for $\widehat{G}_{f}(z)$ and $Q_{2}(z) Q_{1}(z)$ in (24) and (23), respectively. A realization of the inverse of $\widehat{G}_{f}^{-1}(z)$ can be explicitly computed as

$$
\left(\left[\begin{array}{cc}
\widehat{E}_{k} & 0 \\
0 & 0
\end{array}\right],\left[\begin{array}{cc}
\widehat{A}_{k} & \widehat{B}_{k}^{f} \\
\widehat{C}_{k} & \widehat{D}_{k}^{f}
\end{array}\right],\left[\begin{array}{c}
0 \\
I_{m_{f}}
\end{array}\right],\left[\begin{array}{ll}
0 & -I_{m_{f}}
\end{array}\right], 0\right),
$$

while the realization of $\widehat{G}_{f}^{-1}(z) Q_{2}(z) Q_{1}(z)$ results applying straightforward series coupling formulas as

$$
\begin{gathered}
\left(\widetilde{E}_{k}, \widetilde{A}_{k}, \widetilde{B}_{k}, \widetilde{C}_{k}, \widetilde{D}_{k}\right):= \\
\left(\left[\begin{array}{ccc}
\widehat{E}_{k} & 0 & 0 \\
0 & 0 & 0 \\
0 & 0 & \widehat{E}_{k}
\end{array}\right],\left[\begin{array}{ccc}
\widehat{A}_{k} & \widehat{B}_{k}^{f} & 0 \\
\widehat{C}_{k} & \widehat{D}_{k}^{f} & \widehat{C}_{k} \\
0 & 0 & \widehat{A}_{k}
\end{array}\right],\left[\begin{array}{c}
0 \\
\widehat{D}_{k} \\
\widehat{B}_{k}
\end{array}\right],\left[\begin{array}{lll}
0-I_{m_{f}} & 0
\end{array}\right], 0\right)
\end{gathered}
$$

If we apply a Lyapunov similarity transformation with the periodic matrices

$$
W_{k}=\left[\begin{array}{ccc}
I & 0 & I \\
0 & I & 0 \\
0 & 0 & I
\end{array}\right], \quad Z_{k}=\left[\begin{array}{rrr}
I & 0 & -I \\
0 & I & 0 \\
0 & 0 & I
\end{array}\right]
$$

we obtain the transformed system

$$
\begin{gathered}
\left(W_{k} \widetilde{E}_{k} Z_{k+1}, W_{k} \widetilde{A}_{k} Z_{k}, W_{k} \widetilde{B}_{k}, \widetilde{C}_{k} Z_{k}, \widetilde{D}_{k}\right)= \\
\left(\left[\begin{array}{cc|c}
\widehat{E}_{k} & 0 & 0 \\
0 & 0 & 0 \\
\hline 0 & 0 & \widehat{E}_{k}
\end{array}\right],\left[\begin{array}{cc|c}
\widehat{A}_{k} & \widehat{B}_{k}^{f} & 0 \\
\widehat{C}_{k} & \widehat{D}_{k}^{f} & 0 \\
\hline 0 & 0 & \widehat{A}_{k}
\end{array}\right],\left[\begin{array}{c}
\widehat{B}_{k} \\
\widehat{D}_{k} \\
\widehat{B}_{k}
\end{array}\right],\left[0-I_{m_{f}} \mid 0\right], 0\right)
\end{gathered}
$$

with a non-observable trailing part. By removing the nonobservable trailing part, we obtain the realization (25) of $\widehat{G}_{f}^{-1}(z) Q_{2}(z) Q_{1}(z)$.

\section{REFERENCES}

[1] R. A. Meyer and C. S. Burrus, "A unified analysis of multirate and periodically time-varying digital filters," IEEE Trans. Circuits Syst., vol. 22, pp. 162-168, 1975.

[2] O. M. Grasselli and S. Longhi, "Finite zero structure of linear periodic discrete-time systems," Int. J. Systems Sci., vol. 22, pp. 1785-1806, 1991.

[3] J. Gertler, Fault Detection and Diagnosis in Engineering Systems. New York: Marcel Dekker, 1998.

[4] M. Blanke, M. Kinnaert, J. Lunze, and M. Staroswiecki, Diagnosis and Fault-Tolerant Control. Springer-Verlag, Berlin, 2003.

[5] A. Varga, "New computational paradigms in solving fault detection and isolation problems," Annual Reviews in Control, vol. 37, pp. 2542, 2013.

[6] — , "Computation of minimal periodic realizations of transferfunction matrices," IEEE Trans. Automat. Control, vol. 46, pp. 146149, 2004.

[7] A. Varga and P. Van Dooren, "Computational methods for periodic systems - an overview," in Proc. of IFAC Workshop on Periodic Control Systems, Como, Italy, 2001, pp. 171-176.

[8] P. Zhang and S. X. Ding, "Disturbance decoupling in fault detection of linear periodic systems," Automatica, vol. 43, pp. 1410-1417, 2007.

[9] S. Longhi and A. Monteriù, "Fault detection for linear periodic systems using a geometric approach," IEEE Trans. Automat. Control, vol. 54, pp. 1637-1643, 2009.

[10] J. Chen and R. J. Patton, Robust Model-Based Fault Diagnosis for Dynamic Systems. Kluwer Academic Publishers, London, 1999.

[11] S. Longhi and A. Monteriù, "A geometric approach to fault detection and isolation problem for linear periodic systems," in Proc. of CDC'10, Atlanta, USA, 2010.

[12] A. Varga, "A numerically reliable approach for the synthesis of periodic FDI filters," in Proc. of SAFEPROCESS'12, Mexico City, Mexico, 2012, pp. 498-503.

[13] _ _ "Design of fault detection filters for periodic systems," in Proc. of CDC'04, Paradise Island, Bahamas, 2004.

[14] _ - "On computing minimal realizations of periodic descriptor systems," in Proc. of IFAC Workshop on Periodic Control Systems, St. Petersburg, Russia, 2007, pp. 175-180.

[15] — - "On computing normalized coprime factorizations of periodic systems," in Proc. of MTNS'2010, Budapest, Hungary, 2010, pp. $1173-1178$.

[16] S. Bittanti and P. Colaneri, Periodic Systems: Filtering and Control. Springer-Verlag, Berlin, 2009.

[17] P. M. Frank and X. Ding, "Frequency domain approach to optimally robust residual generation and evaluation for model-based fault diagnosis," Automatica, vol. 30, pp. 789-804, 1994.

[18] A. Varga, "On computing periodic coprime factorizations," in Proc. ECC'2009, Budapest, Hungary, 2009, pp. 1359-1364.

[19] — "Computation of minimal order dynamic covers for periodic systems," in Proc. of ECC'07, Kos, Greece, 2007, pp. 1088-1093.

[20] T. Kailath, Linear Systems. Prentice Hall, Englewood Cliffs, N.J., 1980.

[21] A. Varga, "Computation of Kronecker-like forms of periodic matrix pairs," in Proc. of MTNS'04, Leuven, Belgium, 2004.

[22] _ , "Computational issues for linear periodic systems: paradigms, algorithms, open problems," International Journal of Control, vol. 86, pp. 1227-1239, 2013.

[23] , "Computation of Kalman decompositions of periodic systems," European Journal of Control, vol. 10, pp. 1-8, 2004. 